Résumés des conférences et travaux

\title{
Droit comparé dans les sociétés du Proche-Orient ancien
}

\section{Sophie Démare-Lafont}

\section{(2) OpenEdition \\ Journals}

Édition électronique

URL : https://journals.openedition.org/ashp/615

DOI : 10.4000/ashp.615

ISSN : 1969-6310

Éditeur

Publications de l'École Pratique des Hautes Études

\section{Édition imprimée}

Date de publication : 1 octobre 2009

Pagination : 11-14

ISSN : 0766-0677

\section{Référence électronique}

Sophie Démare-Lafont, "Droit comparé dans les sociétés du Proche-Orient ancien », Annuaire de I'École pratique des hautes études (EPHE), Section des sciences historiques et philologiques [En ligne], 140 | 2009, mis en ligne le 02 octobre 2009, consulté le 06 juillet 2021. URL : http:// journals.openedition.org/ashp/615; DOI : https://doi.org/10.4000/ashp.615 


\title{
DROIT COMPARÉ DANS LES SOCIÉTÉS DU PROCHE-ORIENT ANCIEN
}

\author{
Directeur d'études : $\mathrm{M}^{\mathrm{me}}$ Sophie Démare-Lafont
}

Programme de l'année 2007-2008 : I. Essai de géographie juridique mésopotamienne (suite). - II. Les formulaires juridiques des textes de Nuzi.

Le séminaire de cette année a continué l'examen des formulaires juridiques des contrats mésopotamiens, dans le prolongement des enseignements des années précédentes. Les textes choisis cette année portaient sur le mariage.

L'introduction du cours s'est attachée au style de rédaction des codes, pour mesurer à quel point le mode d'énonciation de la loi diffère du langage des notaires qui rédigent les contrats. À beaucoup d'égards, les textes législatifs ressemblent aux textes scientifiques, ainsi que l'avait remarqué Jean Bottéro. Comme les présages ou les diagnostics médicaux, les lois sont compilées dans des recueils qui les systématisent et les mettent en ordre. La forme casuistique, typique de tous les documents scientifiques, reflète la démarche pragmatique des juristes de chancellerie qui, comme les spécialistes de médecine ou de divination, privilégient l'observation sans pour autant ignorer le raisonnement. Ce propos introductif a été illustré par des extraits de la série de présages Šumma alu, de pronostics médicaux (R. Labat, Traité akkadien de diagnostics et de pronostics médicaux, Paris, 1951), de recueils législatifs (Code de Hammurabi, Lois d'Ešnunna, Lois hittites) ainsi que par le rescrit de Samsu-iluna.

Le formulaire du mariage a été examiné tout d'abord dans la documentation paléoassyrienne. Le verbe-clé est ici ahāzum, « prendre », ayant le mari pour sujet et la femme pour objet. Les clauses des contrats envisagent souvent la question d'une seconde épouse, acceptée ou refusée selon les endroits. La bigamie assyrienne doit être comprise dans le contexte du commerce à longue distance : les marchands établissent deux foyers distincts, l'un en Anatolie et l'autre dans la capitale; les deux familles ne sont jamais appelées à se rencontrer, comme le laisse supposer la description des parcours autorisés à la seconde femme (I 490 : 4-8). Dans ICK I 3 : 3-5, il est permis au mari d'épouser une qadištum à Aššur mais on lui refuse toute autre femme en Anatolie. De même en AKT I 77 : 7-9 est-il interdit au mari d'épouser une qadištum dans deux villes d'Anatolie (le sens du mot qadištum dans ces textes est obscur). Le rang d'épouse secondaire n'a pas d'incidence visible sur le contenu du contrat : ainsi en I 490, les modalités du mariage sont-elles manifestement semblables pour l'épouse principale (aššatum) installée à Aššur (1. 16-18), et l'autre femme anatolienne, désignée comme amtum (1. 3), litt. " servante ». L'emploi de ce second terme n'est pas constant et ne traduit pas un rang ou un statut juridique inférieurs ni même un mode de vie différent : même si l'amtum semble souvent mobile, accompagnant son mari dans ses déplacements anatoliens alors que l'aššatum est plutôt fixée à Aššur, les deux termes alternent parfois dans le même texte sans critère logique apparent. Ainsi la 
tablette TPK 1 161a porte-t-elle aux 1. 16-17 : « il conduira sa "servante" où il voudra » alors que l'enveloppe TPK $1161 \mathrm{~b}$ indique aux 1. 18-20 « il conduira son épouse où il voudra $»$.

La stérilité autorise l'achat d'une esclave par la femme (ICK I 3 : 7-16) ou par le mari, qui dans ce cas peut l'épouser (I 490 : 21-22). Les clauses sur le divorce témoignent d'une remarquable égalité entre les époux, qui sont soumis au paiement de la même somme, en général cinq mines d'argent (I 490, ICK I 3, AKT I 76), parfois deux mines (AKT I 77). Un contrat énonce même une communauté de biens entre les époux (KTS 2 6), le couple étant conjointement propriétaire du domicile conjugal, qui sera partagé entre eux en cas de divorce. Ce type de situation rappelle le cas du remariage de la veuve évoqué dans les Lois assyriennes (tabl. A § 35). Quelques tablettes documentent une situation qui ressemble au mariage inchoatif. Ainsi en TC 67 l'époux doit-il se présenter dans les deux mois de l'engagement matrimonial, sous peine de le rendre caduc. La rupture du mariage inchoatif entraîne le paiement d'un dédommagement, évalué à 10 sicles $1 / 2$ (I 513 1. 6-10 «ils ont terminé leur affaire et L. a payé 10 sicles $1 / 2$ d'argent à A. et I. »). De même en TPK 1 161a est-il question d'une somme d'argent qualifiée de «prix » (šimum, 1. 2) et versée par le mari à la mère de l'épouse. Il y a là sans doute une allusion à ce que les Babyloniens appellent terhatum et qui scelle l'avant-projet de mariage. En l'occurrence, la famille de la femme s'engage à ne pas contester (tuārum, litt. « revenir ») l'engagement qui a été pris. Il semble que la future mariée recevait à cette occasion un voile. Telle est du moins l'interprétation a contrario qu'autorise le texte AKT I 77 : à propos de l'épouse, le texte indique (1. 3-4) que « sa tête est découverte » et un peu plus loin (1. 18) que « son prix n'est pas payé ».

Les contrats paléo-babyloniens, qui ont été étudiés ensuite, utilisent un vocabulaire partiellement identique mais présentent des différences notables avec le corpus paléoassyrien. Ainsi que l'a montré R. Westbrook (Old Babylonian Marriage Law, 1988 [AfO Beih. 23], p. 10 sqq.), l'expression désignant le mariage est ana aššūtim u mutūtim ahāzum, «prendre en qualité d'épouse et de mari », décalque akkadien de la formule sumérienne nam-dam-šè tuku « prendre en qualité de conjoint ». Tel est le cas par exemple dans CT 4856 et BAP 90 (Sippar) ou encore dans BE 6/2 40 et 48 (Nippur). Le contrat CT 4850 (Sippar) présente la forme abrégée ana aššùtim nadānum, « donner en qualité d'épouse ». Lorsque le mariage est seulement envisagé mais que l'identité du futur conjoint est encore incertaine, on utilise la phrase ana aššatam ahāzum, «prendre pour épouse » (e. g. CT $637 \mathrm{~b}: 5$, à propos d'un fils qui reçoit un champ kìma dam ahāzim, « en vue de prendre femme »), ou encore ana mutim nadānum, « donner à un mari » (e. g. CT $4740: 15$ pour une adoption matrimoniale réalisée par une religieusenadītum, qui s'oblige à marier l'adoptée). Le projet matrimonial est ainsi impersonnel et anonyme, à la différence du mariage effectif, personnalisé par les noms des époux. Cette conclusion est soutenue par un document de Nippur (SAOC 44 34) dans lequel est conclu un accord matrimonial devant témoins : la mère et le (beau?-)père de la jeune fille, nommément cités, sont dits accepter le statut de gendre d'un individu identifié par le seul nom de son père. Treize témoins sont énumérés et participent à une cérémonie festive au cours de laquelle ils boivent de la bière et s'oignent d'huile. Il s'agit peut-être là du kirrum évoqué au $§ 27$ des Lois d'Ešnunna. 
Les contrats paléo-babyloniens obéissent en général au schéma suivant : en tête du document figurent les noms des parties et l'une des formules présentées plus haut; puis vient éventuellement la mention du versement de la terhatum ou de la dot, dont la composition est parfois détaillée dans la tablette (e. g. CT 4850 : 6-10), et la clause sur le divorce, le plus souvent inégalitaire puisque la répudiation par le mari donne lieu à une indemnisation de la femme (e. g. CT $4850: 28$, BAP $90: 20-21$, CT $4856: 11$ ) tandis que l'épouse est passible de mort par noyade (BAP $90: 15-16$, CT $4850: 23-24$ ) ou par défenestration (e. g. CT $4856: 15-16$ ), ou de réduction en servitude (BE 6/2 58 : 15-16). Dans de très rares cas, les deux époux s'exposent à la même sanction financière (BE 6/2 40 : 10-11 et 15-16). Le contrat contient parfois un serment et s'achève par une liste de témoins et une date. Un cas particulier est représenté par le contrat de Nippur BE 6/2 48 : juste après la clause initiale sur le mariage (1. 1-3), il est indiqué que la mari a fait adopter ses trois fils par sa femme (1. 4-6); ensuite est évoqué le partage du patrimoine du mari entre ses fils (1. 7-12), puis viennent les dispositions en cas de divorce (1. 13-14 et 15-16) ou de rupture du lien adoptif par les enfants (1. 17-20) ou par leur mère (1. 21-24); enfin sont prévues les modalités de l'entretien de la mère adoptive par ses beaux-fils, qui conditionnent leur vocation successorale (1. 27-32). Il est intéressant de relever que, en Babylonie, l'entretien du conjoint survivant est assuré par le moyen de l'adoption, alors que dans les régions situées plus au nord, en particulier chez les Assyriens, le même résultat est obtenu en transformant l'épouse en «père et mère de la maison ». En Mésopotamie, le sort de la veuve dépend donc des dispositions que son mari voudra bien prendre.

Pour conclure ce dossier paléo-babylonien, on s'est intéressé aux cas de bigamie, en particulier pour les religieuses-nadītum de Marduk (§ 144-147 Code de Hammurabi : TIM 4 47), qui ne peuvent pas porter d'enfant. Les actes de la pratique montrent que l'épouse secondaire est la « servante » ou la « sœur » de l'épouse principale (ce qui ne traduit pas toujours une parenté par le sang), la différence entre les deux statuts étant sans doute liée aux droits successoraux des enfants (R. Westbrook, op.cit., p. 106).

Pour l'époque moyenne, les contrats de mariage sont beaucoup moins nombreux. Seuls deux textes médio-assyriens abordent la question matrimoniale, dans des contextes atypiques. KAJ 7 tout d'abord, concerne une Assyrienne qui avait été asservie pendant une période de famine (cf. KAJ 167 : 4 ana balātu u leqe, « pour rester en vie et être acquise »), puis rachetée et remplacée par une esclave étrangère pour être épousée par son acquéreur. Cette situation rappelle le $\S 39$ des Lois assyriennes tabl. A, où une jeune fille a été « sauvée d'une catastrophe » (ina lumne ballutat), autrement dit vendue par son père pour la maintenir en vie. Le second contrat, TIM 4 45, semble concerner une veuve sans fils ni beau-père, libre de négocier et de contracter son mariage. Les deux époux sont dans une stricte relation d'égalité : ils se doivent mutuellement l'entretien (1. 7-9, « À la campagne comme dans la ville, chacun entretiendra l'autre ») et s'exposent à la même indemnité de divorce d'une demi-mine d'argent (1. 10-16). On retrouve là un trait déjà rencontré dans les contrats paléo-assyriens, ce qui pourrait indiquer que le droit commun d'Aššur reconnaît l'égalité entre époux. Mais le manque de parallèles pour l'époque médio-assyrienne incite à ne pas tirer de conclusion trop hâtive à partir de ce seul contrat.

En Babylonie, le corpus est surtout constitué de tuppi zununnê, « tablettes d'entretien », énumérant les biens donnés par la famille de la mariée à celle de l'homme. 
La justification de ces versements, effectués pendant une durée déterminée (de 7 à 15 ans), est incertaine. Ils pourraient témoigner d'une forme de mariage par « entrée » (erēbu), dans lequel l'époux entre dans la maison de sa belle-famille en qualité de fils (cf. O. Gurney, The Middle Babylonian Legal and Economic Texts from Ur, 1983, p. 136-138). Mais on ne comprend pas pourquoi certains bénéficiaires sont des parents éloignés. On a étudié à titre d'exemple le texte Iraq $11 \mathrm{n}^{\circ} 5$, qui énumère des denrées (orge, huile, farine, moutons), de la laine et des vêtements, le tout pour une valeur de neuf sicles d'or et trois sicles d'argent (1. 26-27). Le seul document de mariage (Iraq $11 \mathrm{n}^{\circ} 4$ ) au sens propre concerne les mêmes protagonistes et s'intitule tuppi ahu$z a t i$, «tablette de mariage » (1. 1). Il contient une liste de biens qui n'est pas exactement semblable à la précédente, puisque la valeur totale est de dix-huit sicles d'or (1. 44) et que les noms cités ne correspondent pas toujours. L'hypothèse du CAD (s. v. zunnunû) selon laquelle les deux types de documents sont de même nature juridique, mais expriment respectivement le transfert du point de vue du marié (zununnû) ou du beau-père (ahuzatu) ne peut être vérifiée, faute de comparaison avec d'autres tablettes matrimoniales. Il est dès lors très difficile de se représenter les modalités de conclusion du mariage dans la Babylonie cassite.

On s'est intéressé pour finir aux quelques documents matrimoniaux d'Emar. D'une manière générale, il est souvent difficile d'isoler le mariage comme catégorie juridique, car le corpus du droit de la famille d'Emar reflète des montages familiaux complexes, élaborés en fonction de nécessités patrimoniales. L'adoption et le mariage sont souvent des supports juridiques permettant d'organiser les rapports économiques entre les individus (e.g. lorsque le mariage est lié à une dette rachetée par le créancier ou par un tiers, cf. E 16 ou RE 63). Il n'existe qu'une seule tablette de tradition dite syrienne (RE 61), qui reprend le formulaire paléo-babylonien du mariage ana aššùti nadānu, « donner en qualité d'épouse » (1. 5-6) et de l'adoption matrimoniale puisque la fille du mari est donnée à l'épouse ana é-gi-a $u$ dumu-munus- $t i$, « comme belle-fille et fille» (1. 10). En revanche, seule la répudiation par le mari est envisagée (1. 18-22) et dans des conditions énigmatiques puisque l'homme devra alors la quitter « comme une ressortissante d'Emar qu'elle est » (1. 21-22, kìma marāt Emar šìt). Les autres contrats appartiennent à la tradition dite syro-hittite mais ne présentent aucune homogénéité de formulaire. La jeune fille est donnée « en qualité de belle-fille » (ana kallūti) dans RE 76 : 6 (cf. aussi RE $6: 3-4$ ) afin d'être donnée ensuite ana aššūti « comme épouse » à un individu (RE $76: 9-11)$. En revanche, c'est la femme qui « fait de NP son mari» dans E 124 : 2 (ana lú mutiši epūš). À l'intérieur de ces contrats, les arrangements familiaux sont très variables : parfois l'épouse doit entretenir son beau-père (RE 6 : 4-8), ou bien elle donne à son propre mari l'une de ses filles en mariage (E 124 : 5-6 et 8-9). Il est donc là encore difficile de se faire une idée de l'institution standard, tant les situations décrites semblent contingentes. 